\title{
ALGORITMO DE ENCAMINAMIENTO PARA UNA RED DE ROUTERS BASADO EN ALGORITMOS GENÉTICOS PARA MEJORAR LA VELOCIDAD DE TRASMISIÓN EN REDES MULTICAST
}

\author{
ALGORITHM FOR ROUTING ROUTERS NETWORK BASED ON GENETIC \\ ALGORITHMS TO IMPROVE SPEED TRANSMISSION NETWORK MULTICAST
}

${ }^{1}$ Oscar John Vera Ramírez, ${ }^{2}$ Carlos Alberto Silva Delgado, ${ }^{2}$ Edith Elizabeth Alfaro Gonzales, ${ }^{3}$ Evelyn Fajardo Espinoza

\section{RESUMEN}

Se describe el desarrollo de un algoritmo de encaminamiento para una red de routers basado en algoritmos genéticos para una red multicast. El algoritmo, surge como una solución a la necesidad de un algoritmo alternativo a los dados por estándares basados en números de saltos o en características propias del medio. El algoritmo proporcionará una ruta alternativa a las dadas por los estándares RIP e OSPF, para ser aplicados en una red multicast.

Palabras clave: Algoritmos genéticos, enrutamiento, multicast, multiobjetivo, Pareto.

\section{ABSTRACT}

The development of a routing algorithm for a network of routers based on genetic algorithms for a multicast network is described. The algorithm emerges as a solution to the need for an alternative standard dice based on hop count or algorithm characteristics of the médium. The algorithm provides an alternative to those given by the RIP and OSPF route standards to be applied in a multicast network

Keywords: genetic algorithms, routing, multicast, multi-objective Pareto.

\section{INTRODUCCIÓN}

Para obtener una mejor utilización de la red, es necesario tener un control explícito sobre los caminos que los datos deben atravesar. Entonces, el flujo total de datos sobre la red puede ser acomodado de forma a minimizar los recursos utilizados, maximizar la probabilidad de enrutamiento de toda la demanda de tráfico y minimizar el costo que ello implica, considerando los parámetros de QoS que cada demanda de tráfico solicita (Stallings, 2001).

Una manera muy utilizada a la fecha de seleccionar explícitamente los caminos es a través de MPLS (MultiProtocol Label Switching). Una red MPLS consiste en un conjunto de nodos llamados Label Switch Routers (LSR), que tienen la capacidad de enrutar paquetes utilizando etiquetas, las cuales son adheridas a cada paquete.

Las etiquetas definen un flujo de paquetes entre dos nodos de la red, o, en el caso de una transmisión punto a multipunto, entre el nodo origen y el conjunto de nodos destinos. Por cada flujo, denominado Forwarding Equivalent Class (FEC), un camino específico (Label Switch Path LSP) es definido a través de la red. La Figura 1 muestra un dominio MPLS, el cual puede representar un sistema autónomo (Autonomous System - AS) administrado por alguna entidad privada, como ser un proveedor de servicios de Internet (Internet Service Provider - ISP) (Stallings, 2001). (Ver figura 1).

\section{MATERIALES Y MÉTODOS}

\section{Población}

Debido a que la población es pequeña, dos redes en las cuales probar el sistema (simulado), se ha considerado todo el universo, en consecuencia se toma a toda la población.

\section{Técnicas e instrumentos}

Se utilizó la observación como una técnica de reco-

${ }^{1}$ Doctor en Mecatrónica, Ingeniero Electrónico. Docente de la Facultad de Ingeniería de la Universidad Nacional de Moquegua. Moquegua-Perú. ${ }^{2}$ Ingeniero en Informática y Sistemas. Docente de la Facultad de Ingeniería de la Universidad Nacional de Moquegua. Moquegua-Perú.

${ }^{3}$ Administradora de Empresas. Universidad Nacional Jorge Basadre Grohmann. Tacna-Perú. 
Vera, O. et al. Diseño e implementación de un algoritmo de encaminamiento para una red de routers basado en algoritmos genéticos.

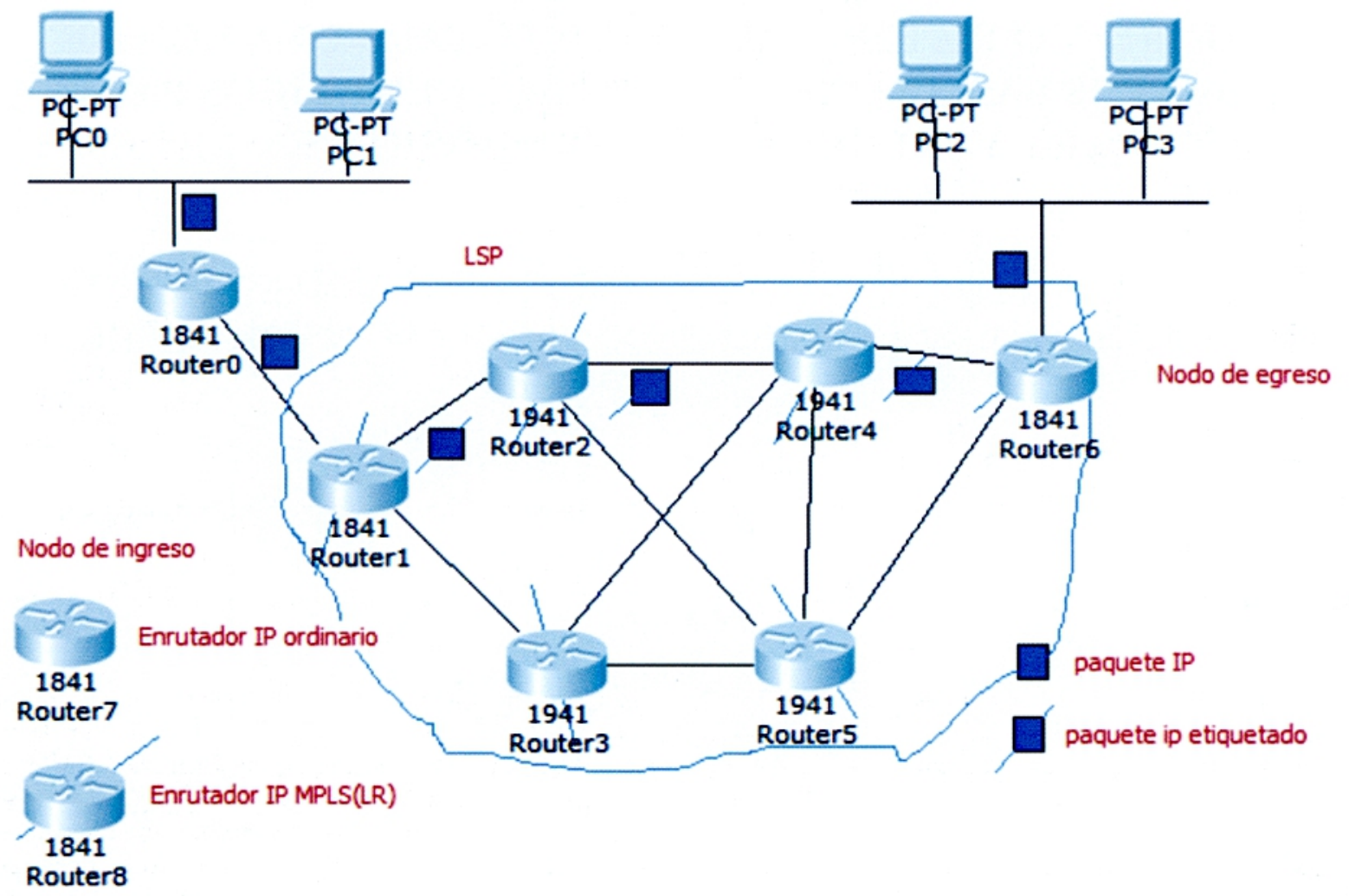

Figura 1. Dominio de LPS

pilación de datos semi - primaria, y con el apoyo de una guía de observación de campo.

\section{Plan de Recolección de Datos} de datos:

Se siguieron los siguientes pasos para la recolección

Creación del algoritmo para los métodos MMA1 y MMA2

Obtención de parámetros en simulador.

Obtención de medidas correspondientes

\section{Recursos Computacionales y de Hardware}

Hardware:

Fabricante del sistema: INTEL

Mainboard: INTELD845PESV

Tipo de sistema: X86-based PC

Procesador: x86 Family 15 Model 2 Stepping 7

GenuineIntel $\sim 2399 \mathrm{MHZ}$

Memoria física total: $256,00 \mathrm{MB}$

Memoria virtual total: $881,22 \mathrm{MB}$

Memoria virtual disponible: 469,97 MB

\section{Routers 2620 cisco}

Hardware:

Processor board ID JAD05190MTZ (4292891495).

M860 processor: part number 0, mask 49

1 FastEthernet/IEEE 802.3 interface(s)

2 Low-speed serial (sync/async) network interface(s)

$32 \mathrm{~K}$ bytes of non-volatile configuration memory.

$63488 \mathrm{~K}$ bytes of ATA CompactFlash (Read/Write)
Software 1:

Bridging software.

X.25 software, Version 3.0.0.

Cisco Internetwork Operating System Software

IOS (tm) C2600 Software (C2600-I-M), Version 12.2(28), RELEASESOFTWARE (fc5)

Software 2:

Sistema Operativo: Microsoft Windows XP Professional MATLAB Versión: 7.0 (R14).

Microsoft Excel Versión: 2002 (10.2614.2625)

CISCO PACKET TRACERT 6.0

\section{RESULTADOS}

\section{Problemas de prueba estáticos}

\section{Problema de Prueba 1}

El primer problema de prueba fue tomado de (Zhengying et al., 2001; Araujo y Barbosa, 2002). Sea G (V, E) la red mostrada en la figura 2 , que consiste de 15 nodos y 44 enlaces no dirigidos. Cada enlace (i, j) tiene asociado un costo $c_{i i} y$ un retardo $d_{i j}$ en $m s$, en este orden. Dado el grupo conformado por $\mathrm{s}=1 \mathrm{y} \mathrm{N}=\{2,9,10,13,14\}$, se desea hallar el árbol multicast de costo mínimo $\mathrm{C}_{\mathrm{T}}$, sujeto a $\mathrm{D}_{\mathrm{M}}<26 \mathrm{~ms}$. En este trabajo extendemos la formulación del problema a uno multiobjetivo, donde los objetivos a optimizar son: 1- $\mathrm{C}_{\mathrm{T}}, 2-\mathrm{D}_{\mathrm{M}} \mathrm{y}$ 3-D $\mathrm{D}_{\mathrm{A}} \cdot \mathrm{C}_{\mathrm{T}}$ se calcula como la suma de los costos de los enlaces. De esta forma, la restricción de retardo ha sido formulada explícitamente como una nueva función objetivo, pero con una función objetivo más: retardo máximo de extremo a 


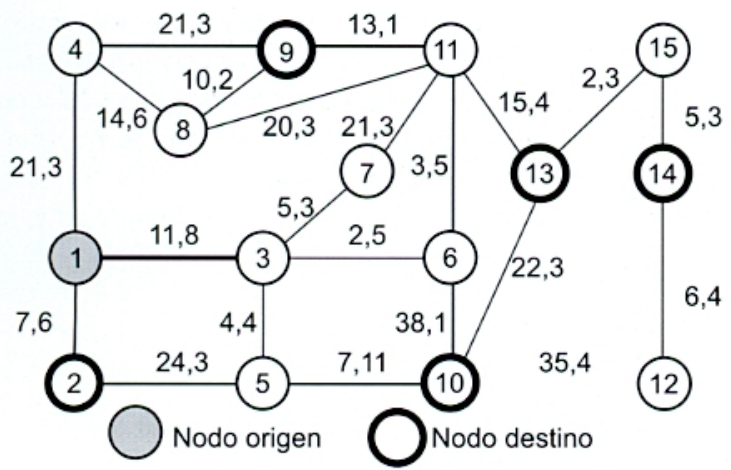

Figura 2. Problema de prueba 1.

extremo. A pesar de la nueva función objetivo. Este conjunto fue hallado por búsqueda exhaustiva en un tiempo de corrida de aproximadamente 20 minutos (Crichigno y Barán, 2004b). El frente Pareto correspondiente es mostrado en la figura 3.

Para este problema de prueba, 100 corridas fueron realizadas. Los algoritmos pararon al ocurrir uno de los siguientes eventos: 1- el tiempo de corrida alcanzó los 150 $\mathrm{ms}$, o 2- se han encontrado las 7 soluciones óptimas. El tamaño de la población fue de 40 individuos para MMA1 y 25 individuos para MMA2. La probabilidad de mutación de MMA1 fue de 0,4. MMA2 halló el conjunto Pareto en las 100 corridas, en un tiempo medio de $22,7 \mathrm{~ms}$.

La corrida de mayor duración fue de $50 \mathrm{~ms}$ y la desviación estándar de $11,4 \mathrm{~ms}$. Por su parte, MMA1 no pudo hallar el conjunto Pareto en todas las corridas. En algunas, solo obtuvo parte de dicho conjunto. La tabla 1 muestra la frecuencia de soluciones Pareto halladas por MMA1. En el peor de los casos (8 corridas), 3 soluciones óptimas fueron halladas, mientras que en el mejor (47 corridas), todas las soluciones del conjunto Pareto fueron obtenidas.

Tabla 1. Frecuencia de soluciones halladas por MMA1

\begin{tabular}{llllll}
\hline Soluciones halladas & 3 & 4 & 5 & 6 & 7 \\
$\mathrm{~N}^{\circ}$ corridas & 8 & 22 & 17 & 6 & 47 \\
\hline
\end{tabular}

Los resultados experimentales mostraron que MMA1 halló las soluciones a, b y c en el $100 \%$ de las corridas, y las soluciones a, b, c y d en el $92 \%$ de las corridas. Las soluciones e, f, y g (juntas) solo fueron obtenidas en aquellas corridas donde todas las soluciones óptimas fueron halladas, es decir, en el $47 \%$ de los casos. De la figura 3, se observa que los vectores objetivos del frente Pareto están dispersos unos de otros, dificultando la obtención del conjunto Pareto óptimo. Sin embargo, MMA2 pudo hallar dicho frente en todas las corridas. Esto demuestra una mayor capacidad de exploración de las regiones del espacio por parte de este algoritmo.

A pesar de que solo MMA2 obtuvo el conjunto Pareto en todas las corridas, los resultados de MMA1 aún son satisfactorios, dado que en casi el $50 \%$ de las corridas obtuvo dicho conjunto. Proveer esta amplia variedad de soluciones

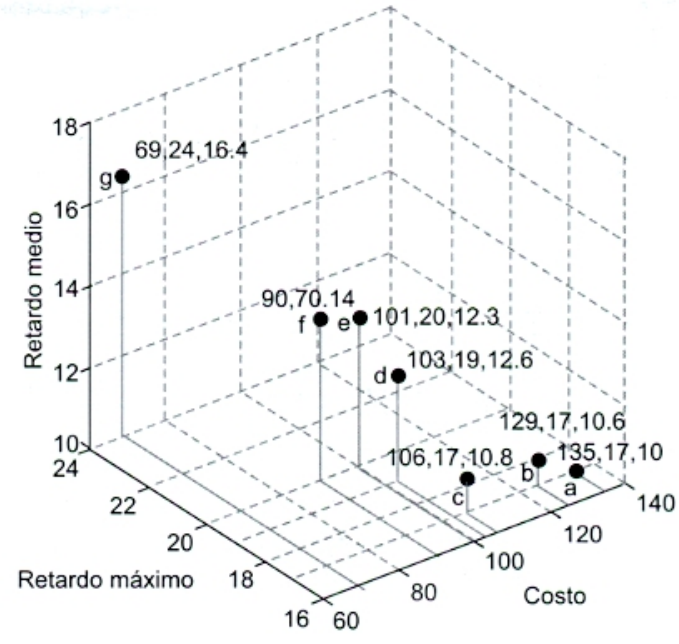

Figura 3. Frente Pareto óptimo del problema de prueba 1.

es de gran importancia, pues, a diferencia de los algoritmos mono-objetivos, la solución más adecuada puede ser elegida para cada ocasión. Como ejemplo, considere un problema en el cual se desee una solución con $\mathrm{D}_{\mathrm{M}}<20 \mathrm{~ms}$ (a priori). En el mejor de los casos, un algoritmo basado en restricciones obtendría la solución d. Sin embargo, este enfoque no permite ver al tomador de decisiones la existencia de las soluciones e y f, las cuales tienen un retardo máximo de $20 \mathrm{~ms}$, solo $1 \mathrm{~ms}$ por arriba de la restricción. Con un enfoque multiobjetivo, dichas soluciones serían proveídas y la solución más adecuada podría ser tomada a posteriori.

\section{Problema de Prueba 2}

El conjunto Pareto óptimo, hallado por búsqueda exhaustiva en un tiempo de corrida de aproximadamente 3 horas (Crichigno y Barán, 2004a), está compuesto por las 16 soluciones de la tabla 2. El frente Pareto.

Tabla 2. Conjunto Pareto óptimo del problema de prueba 2.

\begin{tabular}{lll}
\hline & \multicolumn{1}{c}{ Vector Objetivo } & Árbol \\
\hline a 0,$73 ; 8 ; 23 ; 16,8$ & $(5,4) ;(4,2) ;(2,0) ;(4,10) ;(5,6) ;(6,9) ;(9,13)$ \\
b 0,$73 ; 7 ; 36 ; 19,6$ & $(5,4) ;(4,10) ;(10,12) ;(12,13) ;(4,2)(2,0)(5,6)(6,9)$ \\
c 0,$73 ; 6,8 ; 36 ; 23,8$ & $(5,4) ;(4,2)(2,0)(4,10)(10,11)(11,9)(10,12)(12,13)$ \\
d 0,$6 ; 6,4 ; 40 ; 23$ & $(5,4) ;(4,10)(10,12)(12,13)(10,3)(3,0)(6,9)$ \\
e 0,$66 ; 8,4 ; 36 ; 19,4$ & $(5,4) ;(4,10)(5,6)(6,1)(1,0)(6,9)(9,13)$ \\
f 0,$6 ; 9,4 ; 36 ; 21$ & $(5,4) ;(4,10)(5,6)(6,1)(1,0)(6,9)(9,8)(8,12)(12,13)$ \\
g 0,$6 ; 7,4 ; 36 ; 22,2$ & $(5,4) ;(4,10)(10,12)(12,13)(5,6)(6,9)(6,1)(1,0)$ \\
h 0,$53 ; 10,6 ; 38 ; 26,8(5,4) ;(4,10)(10,11)(11,9)(4,2)(2,7)(7,13)(5,6)(6,1)(1,0)$ \\
i 0,$53 ; 9,4 ; 40 ; 27,6$ & $(5,4) ;(4,10)(10,3)(3,0)(10,11)(11,9)(4,2)(2,7)(7,13)$ \\
j 0,$6 ; 8,4 ; 40 ; 21,8$ & $(5,4) ;(4,10)(10,3)(3,0)(5,6)(6,9)(9,8)(8,12)(12,13)$ \\
k 0,$66 ; 7,4 ; 40 ; 20,2$ & $(5,4) ;(4,10)(10,3)(3,0)(5,6)(6,9)(9,13)$ \\
l 0,$6 ; 6,2 ; 40 ; 27,2$ & $(5,4) ;(4,10)(10,3)(3,0)(10,11)(11,9)(10,12)(12,13)$ \\
m 0,$53 ; 8,2 ; 51 ; 30,2$ & $(5,4) ;(4,10)(10,3)(3,0)(10,11)(11,9)(9,8)(8,12)(12,13)$ \\
n 0,$66 ; 6 ; 44 ; 29$ & $(5,4) ;(4,10)(10,3)(3,0)(10,12)(12,13)(13,9)$ \\
o 0,$73 ; 5,8 ; 63 ; 40$ & $(5,4) ;(4,2)(2,0)(0,3)(3,10)(10,11)(11,9)(10,12)(12,13)$ \\
p 0,$73 ; 5,6 ; 71 ; 41,8$ & $(5,4) ;(4,2)(2,0)(0,3)(3,10)(10,12)(12,13)(13,9)$
\end{tabular}

Para este problema, 100 corridas fueron realizas. Los parámetros de MMA1 fueron: $|\mathrm{P}|=40, \mathrm{R}=30 \mathrm{y} \mathrm{P}_{\text {mut }}=0,4$. El tamaño de la población de MMA2 fue de 25 individuos. 
Vera, $\mathbf{O}$. et al. Diseño e implementación de un algoritmo de encaminamiento para una red de routers basado en algoritmos genéticos.

Las corridas pararon al alcanzar $100 \mathrm{~ms}$.

Las métricas de comparación de este problema son: número máximo $\left(\mathrm{S}_{\max }\right)$, mínimo $\left(\mathrm{S}_{\min }\right)$, promedio $\left(\mathrm{S}_{2}\right)$ y desviación estándar $\left(\sigma_{s}\right)$ de soluciones Pareto óptimas obtenidas en las corridas. Los resultados son presentados en la tabla 3.

Tabla 3. Resultados del problema 2

\begin{tabular}{ccc}
\hline & MMA2 & MMA1 \\
\hline $\mathrm{S}_{\max }$ & 16 & 15 \\
$\mathrm{~S}_{\min }$ & 14 & 14 \\
$\mathrm{~S}_{\mathrm{s}}$ & 15,82 & 14,13 \\
$\sigma_{\mathrm{s}}$ & 0,41 & 0,1 \\
\hline
\end{tabular}

Ambos algoritmos tuvieron un buen rendimiento es este problema de prueba, encontrando al menos 14 soluciones óptimas (87,5\% del conjunto Pareto). MMA1 obtuvo 15 soluciones óptimas en 2 corridas y 14 en 98 oportunidades. Por su parte, MMA2 encontró el conjunto Pareto en 83 ocasiones, 15 soluciones óptimas en 16 corridas y 14 en 1 oportunidad. Es claro que MMA2 tuvo un mejor rendimiento que MMA1.

Una particularidad de MMA1 en estas corridas fue que halló 14 soluciones Pareto óptimas en el $98 \%$ de los casos. Las dos soluciones no obtenidas en estas corridas fueron los árboles (o) y (p). Para clarificar las razones por las cuales no se halló estas soluciones en menos de $100 \mathrm{~ms}$, la figura 4 muestra el frente Pareto del problema en un espacio objetivo donde se ha suprimido la métrica $\alpha_{\mathrm{T}}$, graficándolo en un sistema coordenado considerando las funciones objetivos $C_{T}, D_{M}$ y $D_{A}$ como coordenadas.

Sin embargo, es necesario aclarar que cada vector objetivo está constituido por la n-tupla dada por $\alpha_{\mathrm{T}}, \mathrm{C}_{\mathrm{T}}, \mathrm{D}_{\mathrm{M}} \mathrm{y}$ $\mathrm{D}_{\mathrm{A}}$. Los vectores objetivo (o) y (p) están situados en una "esquina" del espacio objetivo. Es de suponer entonces que la principal dificultad de MMA1 se debe a su capacidad de exploración de aquellas regiones del espacio solución donde podrían encontrarse buenas soluciones.

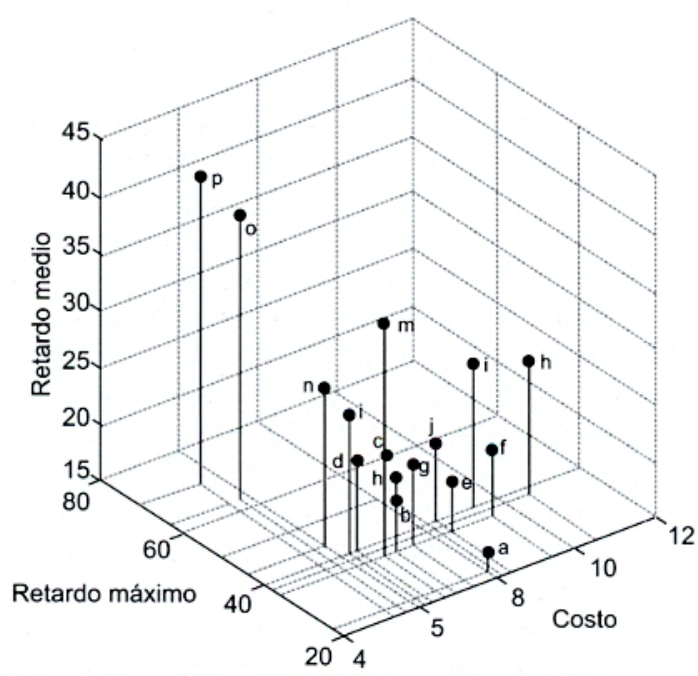

Figura 3. Frente Pareto óptimo del problema de prueba 1.
Dado que ambos algoritmos están basados en el SPEA, y MMA2 no ha tenido el problema citado arriba, es importante apuntar la diferencia entre ambos algoritmos, de forma a entender el motivo de la disparidad de rendimiento. De esto se deriva que el cruzamiento tampoco es el mismo. Por lo tanto, se infiere que la codificación utilizada en MMA2 da a este algoritmo una mayor capacidad de exploración de las regiones del espacio solución. Esta afirmación también fue notada en los problemas dinámicos de prueba presentados en la siguiente sección.

\section{Problemas de Prueba Dinámicos}

\section{Simulaciones Usando la Red de la NSF}

La figura 5 muestra la red de la NSF y la métrica de retardo dij, $¥(i, j) \varepsilon$ E. La capacidad de los enlaces es de 1,5 Mbps.

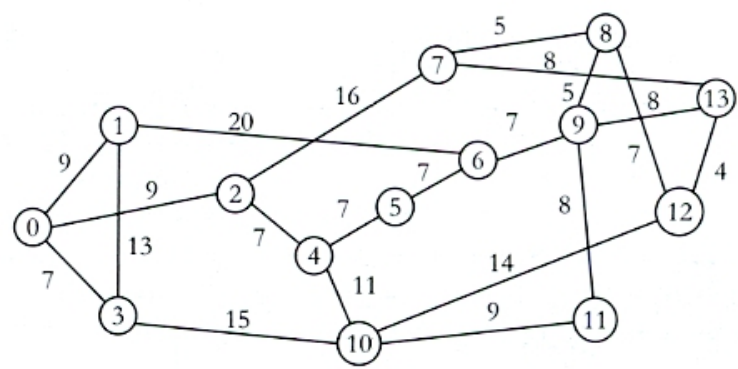

Figura 5. Red de la NFS, con el retardo en ms sobre cada enlace.

Para cada una de las corridas, 300 solicitudes multicast fueron generadas, cada una con una demanda $=150$ Kbps. La tabla 4 muestra los parámetros de MMA1 y MMA2 con los cuales se realizaron las simulaciones, el tiempo medio de cómputo de un árbol multicast (ta) y el rango del tamaño de los grupos multicast. El tiempo medio de cómputo de SK para los distintos valores de $\mathrm{H}$ fue de $1 \mathrm{~ms}$ aproximadamente.

Tabla 4. Parámetros de MMA1 y MMA2, tiempo medio de construcción de un árbol multicast y rango del tamaño de los grupos multicast.

\begin{tabular}{llcccc}
\hline & & $|\mathbf{P}|$ & Generaciones & $\mathrm{t}_{\mathrm{s}}$ & Tamaño Grupo \\
\hline Corrida 1 & MMA1 & 40 & 400 & $60 \mathrm{~ms}$ & {$[3,5]$} \\
& MMA2 & 30 & 50 & $45 \mathrm{~ms}$ & \\
Corrida 2 & MMA1 & 40 & 400 & $90 \mathrm{~ms}$ & {$[6,10]$} \\
& MMA2 & 30 & 50 & $40 \mathrm{~ms}$ & \\
Corrida 3 & MMA1 & 40 & 400 & $60 \mathrm{~ms}$ & {$[3,5]$} \\
& MMA2 & 30 & 50 & $50 \mathrm{~ms}$ & \\
Corrida 4 & MMA1 & 40 & 400 & $90 \mathrm{~ms}$ & {$[6,10]$} \\
& MMA2 & 30 & 50 & $45 \mathrm{~ms}$ & \\
\hline
\end{tabular}

En estas corridas, los tres algoritmos enrutaron todas las solicitudes multicast. La tabla 4 resume los resultados de las corridas 1 y 2

En todas las corridas, MMA1 y MMA2 construyeron un mayor número de árboles que dominaron a los respectivos árboles de $\mathrm{SK}$. Al aumenta $\mathrm{H}$, la diferencia en la métrica 
Vera, O. et al. Diseño e implementación de un algoritmo de encaminamiento para una red de routers basado en algoritmos genéticos.

Tabla 5. Resultados de las corridas 1, 2, 3 y 4.

\begin{tabular}{|c|c|c|c|c|c|c|c|c|c|c|c|c|}
\hline \multirow[b]{2}{*}{$\mathbf{A} 1-\mathbf{A} 2$} & \multicolumn{3}{|c|}{ Corrida 1} & \multicolumn{3}{|c|}{ Corrida 2} & \multicolumn{3}{|c|}{ Corrida 3} & \multicolumn{3}{|c|}{ Corrida 4} \\
\hline & $\mathrm{ND}_{\mathrm{A1}-\hat{\lambda} 2}$ & $\mathrm{ND}_{\mathrm{A2}-\mathrm{A} 1}$ & $I_{A 1-A 2}$ & $\mathrm{ND}_{\mathrm{A} 1-A 2}$ & $\mathrm{ND}_{\mathrm{A2}-\mathrm{A} 1}$ & $I_{A 1-A 2}$ & $\mathrm{ND}_{\mathrm{A} 1-\Lambda 2}$ & $\mathrm{ND}_{A 2-\mathrm{A} 1}$ & $I_{21-A 2}$ & $\mathrm{ND}_{\mathrm{A} 1-\Lambda 2}$ & $\mathrm{ND}_{\hat{A 2}-\mathrm{A} 1}$ & $\mathbf{I}_{\mathrm{A} 1-\mathrm{A} 2}$ \\
\hline MMA1 - MMA2 & 63 & 36 & 201 & 49 & 81 & 170 & 53 & 54 & 193 & 49 & 79 & 172 \\
\hline MMA1-SK $(\mathrm{H}=0)$ & 82 & 26 & 192 & 69 & 13 & 218 & 64 & 33 & 203 & 58 & 16 & 226 \\
\hline MMA1-SK(H=2) & 173 & 19 & 108 & 181 & 14 & 105 & 165 & 23 & 112 & 195 & 13 & 92 \\
\hline MMA1-SK $(\mathrm{H}=4)$ & 243 & 7 & 50 & 226 & 4 & 70 & 236 & 8 & 56 & 233 & 5 & 62 \\
\hline MMA2-SK $(\mathrm{H}=0)$ & 70 & 31 & 199 & 64 & 11 & 225 & 66 & 40 & 194 & 52 & 8 & 240 \\
\hline MMA2-SK $(H=2)$ & 169 & 14 & 117 & 201 & 7 & 92 & 164 & 24 & 112 & 191 & 13 & 96 \\
\hline MMA2-SK $(\mathrm{H}=4)$ & 245 & 11 & 44 & 241 & 4 & 55 & 247 & 11 & 42 & 255 & 2 & 43 \\
\hline
\end{tabular}

de dominancia fue aún mayor a favor de los algoritmos propuestos en este trabajo. En las 4 corridas, MMA1 y MMA2 construyeron mejores árboles que SK $(\mathrm{H}=2)$ y SK $(\mathrm{H}=4)$ en más del $50 \%$ de las solicitudes multicast. Cuando los algoritmos propuestos en este trabajo fueron comparados con SK $(\mathrm{H}=0)$, se observa un gran número de indiferencias.

Esto se debe a la relación de compromiso entre los objetivos. Dado que MMA1 y MMA2 eligen una solución del frente, dicha solución puede no tener las cuatro funciones objetivo menores que la solución de SK, y viceversa, conllevando a una situación donde se presentan conflictos entre los objetivos, y creando las citadas relaciones de compromiso.

De todas maneras, note que NDMMA1-SK $(\mathrm{H}=0)$ es mayor que $\operatorname{NDSK}(\mathrm{H}=0)$-MMA1. De forma similar, NDMMA2-SK $(\mathrm{H}=0)$ también es mayor que $\operatorname{NDSK}(\mathrm{H}=0)$ MMA2, indicando que ambos algoritmos proveyeron un mayor número de soluciones que dominaron a los correspondientes árboles de $\mathrm{SK}(\mathrm{H}=0)$.

Cuando MMA1 y MMA2 son comparados, el aspecto principal a resaltar es que, en modo denso (corridas 2 y 4 ), MMA2 claramente tuvo un mejor comportamiento que MMA1, dado que NDMMA2-MMA1 > NDMMA1-MMA2. En modo esparzo (corridas 1 y 3), MMA1 obtuvo un mayor número de dominancias en la corrida 1 , mientras que en la corrida 3 la dominancia entre ambos algoritmos fue similar.

\section{DISCUSIÓN}

Además de los estudios comparativos (Srinivas y Deb, 1994; Zitzler y Thiele, 1999), SPEA también ha demostrado su buen funcionamiento en la resolución de problemas de ingeniería como el diseño topológico de redes de computadoras (Barán et al., 2003), la programación de bombeo de agua en sistemas de suministro de agua (Sotelo et al., 2002) y la compensación reactiva de potencia (Barán et al., 2001). Por tal motivo, para resolver el problema de enrutamiento multicast en redes de computadoras, en el presente trabajo se proponen dos algoritmos evolutivos multiobjetivos, denominados MMA1 y MMA2 (Multiobjective Multicast Algorithm) basados en el SPEA.

\section{CONCLUSIONES}

Se diseñó un algoritmo de encaminamiento tomando como premisa un enfoque multiobjetivo, con los siguientes parámetros: la utilización máxima de los enlaces del árbol $\alpha T$, el costo $C T$, el retardo máximo de extremo a extremo $D M$ y el retardo medio $D A$.
Se implementó el protocolo diseñado, con dos nuevos algoritmos evolutivos basados en el SPEA: MMA1 y MMA2. Estos algoritmos obtienen un conjunto Pareto óptimo de soluciones en una corrida. Esta característica es de gran importancia, pues la solución más adecuada puede ser elegida para cada caso particular, sin considerar restricciones a priori.

Al evaluar el rendimiento en el problema de prueba 1 (P1), MMA2 convergió al conjunto Pareto en todas las corridas, mientras que en el problema de prueba 2 (P2) halló todas las soluciones óptimas en el $83 \%$ de los casos. Por su parte, MMA1 obtuvo el frente Pareto en el $47 \%$ de las corridas de P1, y en ninguna ocasión halló el conjunto óptimo de P2. Sin embargo, en todas las corridas obtuvo más del $87 \%$ de las soluciones óptimas. El análisis de los resultados experimentales muestra que la representación cromosómica de MMA2 provee a este algoritmo una mayor capacidad de exploración, permitiendo obtener aquellas soluciones Pareto óptimas ubicadas en "esquinas" del espacio de búsqueda que no siempre son halladas por MMA1.

\section{REFERENCIAS BIBLIOGRÁFICAS}

Araujo, P. y Barbosa, M. (2002). Multicast routing with quality of service and traffic engineering requirements in the internet, based on genetic algorithm. Proceedings of the 7th Brazilian Symposium on Neural Networks. Brazil.

Barán B., y R. Sosa. (2000). A New Approach for Antnet Routing, IEEE International Conference on Computer Communication and Networks (ICCCN'2000), USA.

Baran, B., Duarte, S. y Benítez, D. (2003). Telecommunication Network Design with Parallel Multiobjective Evolutionary Algorithm. IFIP/ACM Latin American Networking Conference. Bolivia.

Barán, B., Vallejos, J., Ramos, R. y Fernández, U. (2001). Multiobjective Reactive Power Compensation, Proceedings of the IEEE Transmission and Distribution Conference and Exposition. USA.

Beverly R., y K. Claffy. (2003). Wide-Area IP Multicast Traffic Characterization, IEEE Network, Vol. 17, $\mathrm{N}^{\circ} 1$, pág. $8-15$.

Coello C. (1999). A comprehensive survey of evolutionarybased multiobjective optimization, Knowledge and Information System.

Coello C. (1996). A Empirical Study of Evolutionary Techniques for multi-objective optimization in Engineering Design, Ph.D Thesis, Department of Computer Science, Tulane University, New Orleans, USA. 


\section{Ciencia \& Desarrollo}

Vera, O. et al. Diseño c implementación de un algoritmo de encaminamiento para una red de routers basado en algoritmos genéticos.

Coello C. (2000). EMOO Web Page, A complete list in alphabetical order, Evolutionary Optimization: an International Journal on Internet.

Cohon J. (1978). Multiobjective programming and planning, Academic Press.

Crichigno J., y B. Barán. (2004). Multiobjective Multicast Routing Algorithm for Traffic Engineering, 13th International Conference on Computer Communications and Networks (ICCCN'2004), Chicago - USA.

Crichigno, J. y Barán, B. (2004a). Multiobjective multicast routing algorithm. 11th International Conference on Telecommunications (ICT 2004). Brazil.

Crichigno, J. y Barán, B. (2004b). A multicast routing algorithm using multiobjective optimization. 11th International Conference on Telecommunications (ICT 2004). Brazil.

Darwin C. (1985). On the Origin of Species by Means of Natural Selection, $6^{\circ}$ Ed., Penguin Classics.

Deb K.(1999). Evolutionary algorithms for multi-criterion optimization in engineering design, Proceedings of the Evolutionary Algorithms in Engineering and Computer Science (EUROGEN'99).
Dijkstra E.(1959). A note on two problems in connexion with graphs, Numerische Mathematik, Vol. 1, pág. 269271.

Sotelo, A., Barán, B. y Von Lucken, C. (2002). Multiobjective evolutionary algorithms for pump scheduling optimization. 3rd International Conference on Engineering Computational Technology ECT-2002. Republica Checa.

Srinivas N. y Deb, K. (1994). Multiobjective optimization using non-dominated sorting genetic algorithm. MIT Evolutionary computation, vol. 2, num 3, pp. 221 248.

Stallings, W. (2001). MPLS. The Internet Protocol Journal, vol. 4, num. 3.

Zhengyng, W., Bingxin, S. y Erdun, Z. (2001). Bandwidthdelay-constraint least-cost multicast routing based on heuristic genetic algorithm. Computer comunications, vol24, pag. 685-692.

Zitzler, E. y Thiele, L. (1999). Multiobjective Evolutionary Algorithms: A comparative case study and the strength pareto approach. IEEE Trans. Evolutionary Computation, vol. 3, num. 4, pp. 257-271.

\section{Correspondencia:}

Oscar John Vera Ramirez: programadorfox@hotmail.com
Fecha de Recepción: 28/05/2015

Fecha de Aceptación: 16/09/2015 